\title{
Designing Social Patterns Using Advanced Separation of Concerns
}

\author{
Carla Silva $^{1}$, João Araújo ${ }^{2}$, Ana Moreira $^{2}$, and Jaelson Castro ${ }^{1}$ \\ ${ }^{1}$ Centro de Informática, Universidade Federal de Pernambuco, 50732-970, Recife, Brazil \\ \{ctlls, jbc\}@cin.ufpe.br \\ ${ }^{2}$ Dept. Informática, FCT, Universidade Nova de Lisboa, 2829-516 Caparica, Portugal \\ \{ja, amm\}@di.fct.unl.pt
}

\begin{abstract}
This paper proposes an approach to support separation and modularization of crosscutting concerns in multi-agent systems (MAS). Crosscutting concerns are properties that do not align well with the decomposition criteria of the chosen approach and, therefore, cannot be modularized. Aspect-Oriented Software Development offers mechanisms to encapsulate such properties in separate modules, the aspects. Aspects are used as abstractions to capture social patterns concerns that cut across functional modules in MAS. To achieve this, we propose a technique to describe social patterns in an aspect-oriented context and a systematic way for using them in MAS design.
\end{abstract}

\section{Introduction}

Tropos [1] has defined a set of design patterns, called social patterns [2], focusing on social and intentional features which are recurrent in cooperative and multi-agent systems (MAS). Although both application core and design patterns concerns are independent from each other [3], the current use of patterns can lead to the scattering and tangling of their concerns with the application functional modules. As a consequence, application core becomes dependent on patterns [3], which do not fully meet quality requirements, such as understandability, maintainability, evolvability and reusability. Thus, design patterns concerns can be called crosscutting concerns, since they cut across the functional modules concerns, decreasing the system reusability and maintainability. These crosscutting concerns can be better addressed by adopting aspect-oriented software development (AOSD) techniques [4]. The purpose of this new technology is to localize crosscutting concerns, avoiding their scattering or tangling through software artifacts [5].

Although many languages for multi-agent systems modeling have been proposed in the last few years, such as AUML [6] and MAS-ML [7], they do not consider the separation of crosscutting concerns. On the other hand, some languages for modeling separation of crosscutting concerns have been proposed, such as aSideML [8] and AODM [9], but they do not address MAS directly. In this context, we propose a notation to describe social patterns using abstractions and mechanisms provided by aspect orientation to support the separation of crosscutting concerns in MAS. To achieve this, we need to: (i) specialize the agency metamodel [10] by using the concept of 
model roles [11] used in Pattern Specifications (PSs) technique [12]; (ii) attach notes to the structural model of the social pattern to capture some abstractions of aspect orientation; and (iii) suggest guidelines to map the proposed notation to the constructs of AspectJ [4] and JADE [13] environments. We have used an integration of JADE and AspectJ to implement our approach. This integration is not difficult as both implementation environments are based on Java, facilitating the combination of JADE and AspectJ code in the same program.

This paper is organised as follows: Section 2 presents some background on Social Patterns, UML-based MAS notation and PSs technique. Section 3 presents the crosscutting nature of social patterns through a motivation example. Section 4 introduces our notation to describe social patterns in an aspect-oriented context. Section 5 exemplifies the use of our approach. Section 6 presents some related work. Finally, section 7 summarises our work and points out to future work.

\section{Background}

The purpose of this work is to propose a description of social patterns using advanced separation of concerns. This description is achieved using model roles [11] to specialize the agency metamodel introduced in [10]. The concept of model roles has been used to specialize the UML metamodel [14], in the PSs technique [12], to specify what model elements must participate in the pattern. Thus, in this section we introduce the social patterns, the agent oriented notation and the PSs technique.

\subsection{Social Patterns}

Tropos is a requirements-driven framework aimed at building software that operates within a dynamic environment. To promote an efficient development of MAS, Tropos supports five phases of software development: Early Requirements, Late Requirements, Architectural Design, Detailed Design and Implementation. In this work we will concentrate on the late phases.

The Detailed Design phase is intended to introduce additional detail for each architectural component of a system. Designers can be guided by a catalogue of multiagent patterns which offer a set of standard solutions. In particular, Tropos has defined a set of design patterns, called social patterns [2], focusing on social and intentional aspects that are recurrent in multi-agent and cooperative systems. They are inspired by the federated patterns introduced in [15] [16]. Examples of social patterns are booking, subscription, monitor, broker, mediator, wrapper and matchmaker (to be used to illustrate the approach). The Matchmaker pattern involves an intermediary agent (matchmaker) that receives requests from service Providers to subscribe/unsubscribe its services into the Yellow Pages maintained by it. A Client may need a specific service provided by an unknown Provider. The Matchmaker also receives requests from Clients to locate some Providers which offer a specific service. If there is some Provider for the requested service, the Matchmaker informs that Provider's identification to the Client which, in turn, can directly interact with it [17]. 


\subsection{UML-Based MAS Notation}

In this section, we present the MAS architectural diagram specified according to the agency metamodel introduced in [10] and reflecting the client-server pattern [18] that we have tailored for MAS. We define the MAS architectural diagram (Fig. 1) in terms of AgentRoles and organizational architectural features which include: Goal, MacroPlan, ComplexAction, OrganizationalPort, AgentConnector, Dependum, Dependee and Depender. A Dependum defines an "agreement" of service offer between two agent roles that also play the roles of Depender and Dependee. Thus, the AgentRole responsible for providing the service is the Dependee. The AgentRole that requests the service provided is the Depender. A Dependum can be of four types: goals, softgoals, tasks and resources [19]. AgentRoles need to exchange signals through an AgentConnector to accomplish the contractual agreement of service providing between them. An OrganizationalPort specifies a distinct interaction point between the AgentRole and its environment (depicted as a white square attached to the «AgentRole» class). A Goal is a condition or state of affairs in the world that the actor (Agent or AgentRole) would like to achieve. How the goal is to be achieved is not specified, allowing alternatives to be considered [20]. A MacroPlan encapsulates the recipe for achieving some goal. A ComplexAction determines the steps to perform a MacroPlan.



Fig. 1. MAS Architectural Diagram

For example, in Fig. 1 we have the Provider AgentRole which is responsible for performing the service defined in the Dependum. This AgentRole aims at achieving the ServicePerformed goal by executing the PerformPlan MacroPlan, which, in turn, consists of performing the service() ComplexAction. The Client AgentRole aims at achieving the ServiceRequested goal by executing the RequestPlan MacroPlan, which, in turn, consists of performing the request() ComplexAction. Therefore, the Client AgentRole is responsible for requesting the service defined in the Dependum. Both the message for requesting the service execution and the message for confirming whether the service was successfully concluded are sent through the AgentConnector.

\subsection{Pattern Specifications}

Pattern Specifications are introduced in [12] as a way of formalizing the structural and behavioral features of a pattern. The notation for PSs is based on the Unified 
Modeling Language (UML). The abstract syntax of UML is defined by a UML metamodel [14]. PSs specialize this metamodel by specifying what model elements must participate in the pattern and is defined in terms of roles. Each element in the specification of the patterns is a role, that is, a metaclass specialized by additional properties that any element fulfilling the role must possess. A PS can be instantiated by assigning UML model elements to the roles in the PS. A model conforms to a PS if its model elements that play the roles of the PS satisfy the properties defined by the roles. An example of a PS for class diagram and a conforming UML class diagram is given in Fig. 2. Roles are identified by preceding them with a vertical bar, "l".

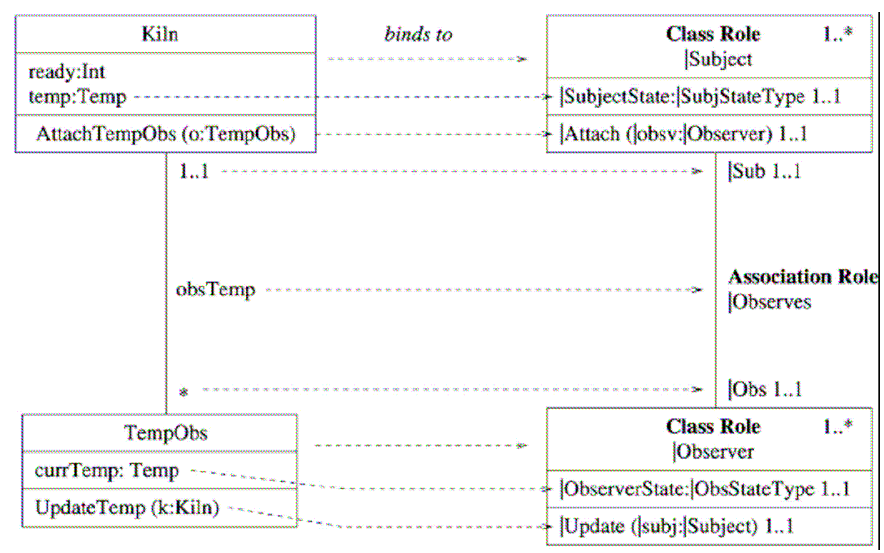

Fig. 2. A PS class diagram (right) and a conforming UML class diagram (left). (From [12])

The PS model is a specialization of the metamodel for UML class diagrams but is presented graphically so that it resembles a class diagram not the metamodel. The right-hand side of Fig. 2 is a PS model defining the structure of a general Observer pattern [21]. The left-hand side shows a UML model that conforms to the PS model Kiln plays the role of ISubject, TempObs plays the role of IObserver, currTemp plays the role of IObserverState, AttachTempObs plays the role of IAttach, and so on. Note that the definition of conformance allows other model elements to appear in the conforming model as long as the original role constraints remain satisfied.

In the next section we present a motivation example for the separation of the social patterns concerns in MAS.

\section{The Crosscutting Nature of the Matchmaker Pattern}

Several design patterns for multi-agent systems have been proposed $[2,15,16]$ to describe recurring problems and solutions in software design and, therefore, improving software reusability and maintainability. However, current software development paradigms cannot avoid that application core becomes dependent on patterns, decreasing possibilities of reuse [3]. In order to show the crosscutting nature of the Matchmaker pattern, let us consider the implementation of each pattern participant concern 
(i.e., Client, Matchmaker and Provider concerns) provided by the JADE's [13] API (Application Programming Interface). JADE is a suitable agent platform to support the implementation of MAS. In JADE, a behavior represents a task that an agent can carry out.

Important features that JADE provides are the ability of agents to communicate with each other and the DF (Directory Facilitator) agent, which implements the behavior of the main participant of the Matchmaker pattern [2] i.e., the Matchmaker agent. It provides a Yellow Pages service by means of which an agent (Client) can find other agents providing the services it requires in order to achieve its goals. An agent wishing to publish one or more services (Provider) must provide the DF with a description including its Agent Id (AID) and the list of its services. The services provided by the DF agent (Matchmaker) are usually used by all agents of a MAS implemented in JADE. Thus, all agents who need to register or unregister its services in the Yellow Pages of the DF agent will implement the Provider's concern (Fig. 3 and Fig. 4).

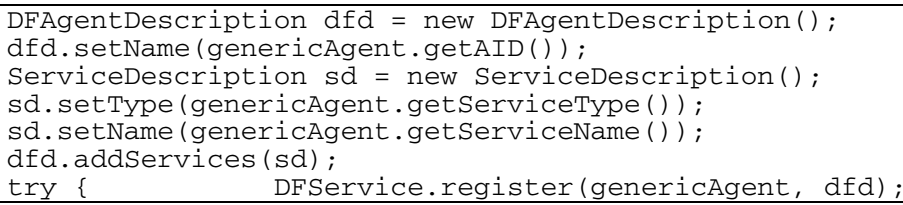

Fig. 3. Code to register the service in the yellow pages

try $\{\quad$ DFService.deregister(genericAgent);

Fig. 4. Code to unregister the service in the yellow pages

Similarly, all agents who need to find a specific provider agent in the Yellow Pages will implement the Client's concern (Fig. 5).

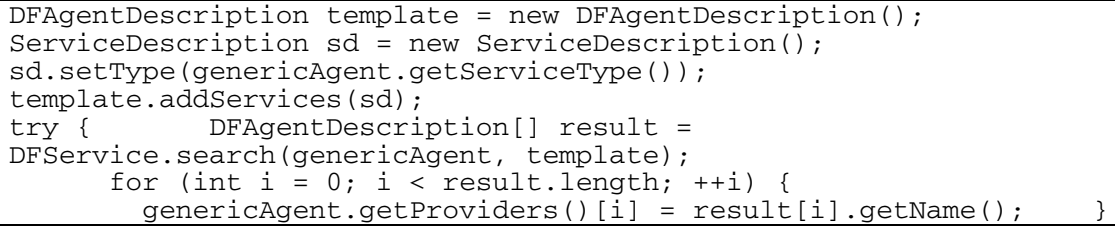

Fig. 5. Code to search in the yellow pages the agent which provides a service

Generally, the Yellow Pages Provider and Yellow Pages Client concerns (henceforth YPProvider and YPClient concerns, respectively) are going to be implemented by several agents involved in the Matchmaker pattern. Consequently, the YPProvider and YPClient concerns become tangled with the concerns of these agents (i.e., application core functionality) and scattered in the system. To solve this issue, we can use aspect-oriented abstractions both in the design and implementation phases. 
Observe that the concern of the Matchmaker participant, i.e. the Yellow Pages service concern, is implemented inside the DF agent and its implementation is hidden in the JADE's API. Thus, the Matchmaker participant concern will not crosscut the application functional modules.

Next section presents a notation to describe social patterns using advanced separation of concerns.

\section{A Standard Technique for Social Pattern Specification}

To describe social patterns we propose an approach that specializes the agency metamodel for MAS architectural diagram [10] by using the concept of model roles [11]. In fact, the concept of model roles was used in the Pattern Specifications technique [12] to specialize the UML metamodel for specifying which model elements must participate in a pattern. The work presented in [22] uses the Pattern Specifications (PS) technique [12] to address aspect modeling. Analogously, we can use PSs to promote the separation of social patterns concerns in MAS design.

The following sub-sections describe the template used to specify a social pattern (section 4.1) and the respective structural specification (section 4.2). These are illustrated by the Matchmaker pattern. The communication specification describes a pattern of communications and can be found in [23].

\subsection{Pattern Template}

The template used to describe social patterns (with a subset of GoF's template [21]) was introduced in [17] to illustrate the Matchmaker pattern. Its main attributes are Name, Intent, Applicability, Motivation example, Problem, Solution and Participants.

\subsection{Structural Agent Pattern Specification}

A structural agent pattern specification (SAPS) defines the part of the pattern metamodel that characterizes MAS architectural diagram views of pattern solutions. It defines subtypes of agency metaclasses describing MAS architectural diagram elements (e.g., agency metaclasses AgentRole, AgentConnector) and specifies semantic pattern properties using constraint templates (see Fig. 6). A SAPS consists of a structure of pattern roles [12], where a role specifies properties that a MAS model element must have if it is to be part of a pattern solution model. Formally, a role defines a subtype of an agency metaclass. The metaclass is called the base of the role. For example, a role that has the metaclass AgentConnector as its base specifies a subset of MAS agent connectors. A MAS model element conforms to (or plays) a role if it satisfies the properties defined in the role, that is, the element is an instance of the subtype defined by the role. A role in a SAPS can be a classifier or a relationship role. A role that has the base Classifier or a base that is a subtype of Classifier (e.g., AgentRole, Dependum) is a classifier role. A relationship role is any role that has the base Relationship or a base that is a subtype of Relationship (e.g., AgentConnector). 


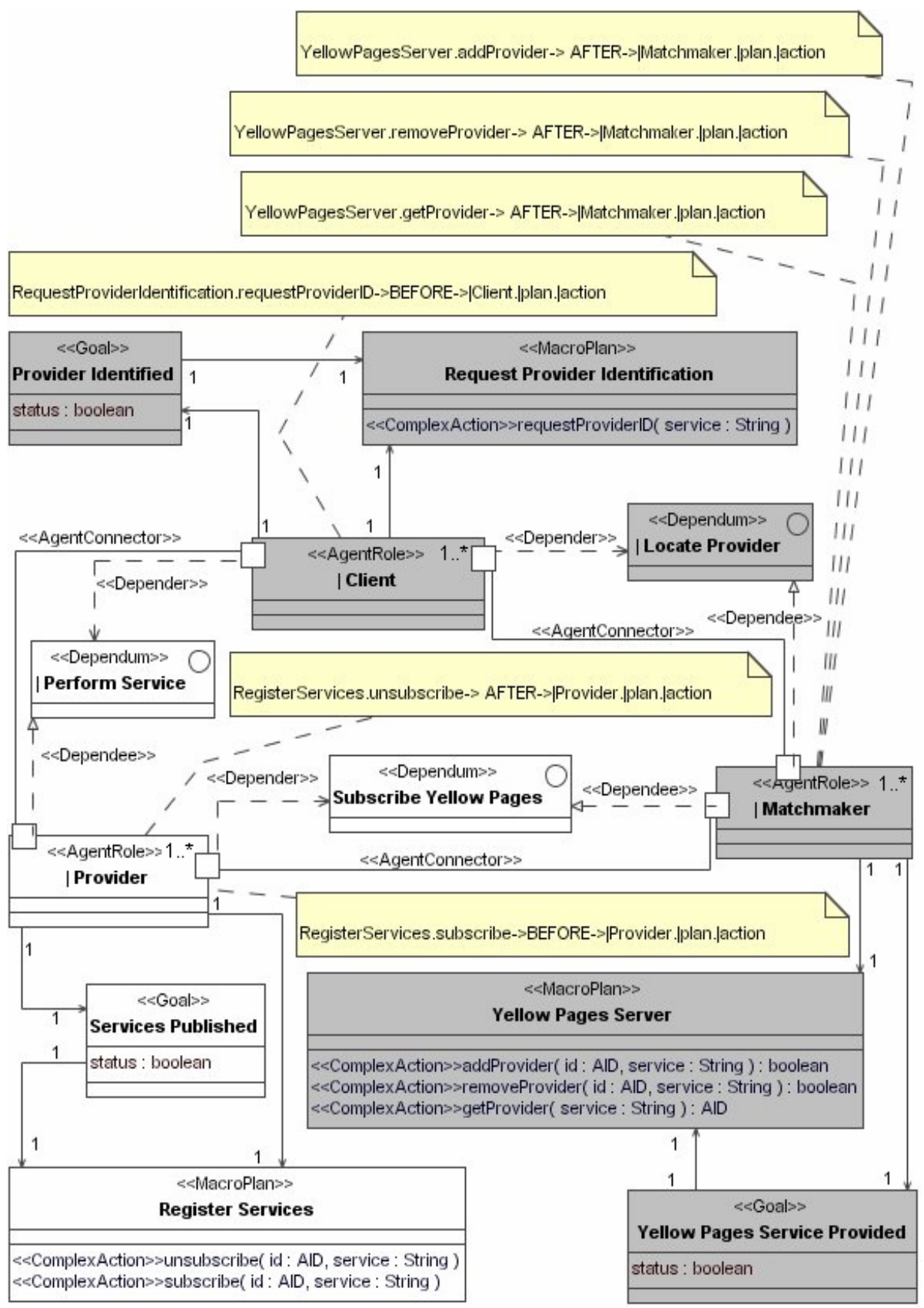

Fig. 6. Matchmaker Structural Agent Pattern Specification

Fig. 6 shows a SAPS that specifies solutions for the Matchmaker pattern [2]. This SAPS is described using the client-server architectural pattern (Fig. 1). The Matchmaker pattern has to provide three services (i.e. dependums): Locate Provider, 
Perform Service and Subscribe Yellow Pages. For example, the shaded area of Fig. 6 represents the classes involved in the ILocate Provider service defined in a Dependum class. When the IClient AgentRole executes the Request Provider Identification MacroPlan by performing the requestProviderID ComplexAction to achieve the Provider Identified Goal, it triggers a request to the IMatchmaker AgentRole to perform the ILocate Provider Service. The IMatchmaker AgentRole performs the requested service because it does not conflict with the achievement of the Yellow Pages Service Provided Goal. A conflict is detected when the service requested to an agent playing a specific agent role, can cause the failure of some of its goals. So, both the requested service and the goal achievement are accomplished by means of the Yellow Pages Server MacroPlan. The description of the IPerform Service and Subscribe Yellow Pages services is achieved in a similar way.

Here we will use the idea proposed in [22] for using model roles only in the elements of the pattern that will vary from one application to another, since when the pattern is applied to a specific application, these roles will be instantiated to model elements of the application. For example, the SAPS in Fig. 6 consists of only five model roles: three for AgentRole metaclass (Client, Provider and Matchmaker) and two for Dependum metaclass (ILocate Provider and IPerform Service). The roles define subtypes (specializations) of metaclasses in the Agency metamodel. For example, the Client role defines a subtype of metaclass AgentRole in the Agency metamodel. The AgentRole roles indicate that conforming architectural diagrams must have at least one AgentRole that conforms to the Client role (as indicated by the $1 .{ }^{*}$ realization multiplicity in the first compartment of the AgentRole role), at least one AgentRole that conforms to the Provider role, and at least one AgentRole that conforms to the Matchmaker role. An AgentRole that conforms to the Matchmaker role (referred to as a Matchmaker class) must have exactly one goal that conforms to the Yellow Pages Service Provided role and exactly one MacroPlan that conforms to the Yellow Pages Server role. The same rationale can be applied to the other roles which are subtypes of metaclass AgentRole in the Agency metamodel.

Moreover, we have the model roles present in the notes which describe the AgentRoles' points affected by the aspects encapsulating the Matchmaker pattern's concerns (each aspect will encapsulate the concern of one pattern participant). For example, let us consider the note attached to the IClient AgentRole. It represents that the execution of the requestProviderID(), which is part of the RequestProviderIdentification, is going to affect the client participant of the Matchmaker pattern before the execution of some of its actions, which is part of some of its plans. To determine who are the client participant in the application and its affected action, we need to instantiate each model role present in the SAPS (e.g., IClient, laction, Iplan, etc.) to design elements present in the MAS architectural design. Thus, we weave the aspects with the AgentRoles resulting in the application of the pattern to the system.

In the sequel we propose some mapping guidelines to enable the implementation of social patterns concerns separately from agents concerns through the integration of JADE and AspectJ implementation environments. 


\subsection{Towards Implementation in JADE and AspectJ}

To implement our approach we use the integration of JADE and AspectJ environments. AspectJ [4] is a practical aspect-oriented extension to the Java programming language. This section provides some guidelines to map our notation to represent MAS (Fig.1) and social patterns (Fig. 6) in constructs of JADE and AspectJ, respectively. In the affected Architectural Diagram: (i) each «MacroPlan» becomes a Behavior in JADE; (ii) each «ComplexAction» becomes a simple method; (iii) each «AgentRole» becomes an Agent in JADE, since JADE does not support the concept of agent roles played by agents; (iv) the goal element is not mapped to any JADE construct, as JADE does not support the implementation of cognitive agents.

In the SAPS: (i) each «MacroPlan» presented in Fig. 6 becomes an Aspect in AspectJ; (ii) each «ComplexAction» becomes an Advice in the aspect it belongs to; (iii) each «AgentRole» is a module affected by an aspect; (iv) each note attached to an AgentRole becomes a Pointcut and also defines the rule to compose the aspect with the agent; (v) the goal is not mapped to any AspectJ construct.

\section{An Example}

To illustrate our approach, we consider the domain of Newspaper Office introduced in [24]. The e-News system (Fig. 7) enables a user to read news by accessing the newspaper website maintained by a Webmaster AgentRole which is responsible for updating the published information. The information to be published is provided by the Chief Editor AgentRole. The Chief Editor AgentRole depends on the Editor AgentRole to have the news of a specific category. For example, an Editor may be responsible for political news, while another one may be responsible for sports news. Each Editor contacts one or many Photographers-Reporters which can find the news of specific categories (e.g., about sport news). The Chief Editor then edits the Editor' news and forwards them to the Webmaster to publish them.

The following sub-sections describe the architectural diagram (section 5.1), the choice and application of social patterns (section 5.2) and the partial AspectJ code (section 5.3) for the e-News system.

\subsection{Architectural Diagram}

We start by proposing the architectural solution for the e-News problem (Fig. 7) by using the MAS architectural pattern (Fig. 1). The e-News system is composed of four AgentRoles: Editor, Webmaster, Chief Editor and Photographer-Reporter. For example, in Fig. 7 the shaded area corresponds to the interaction between the Editor and Photographer-Reporter to achieve the service Produce News Article of Specific Subject. The Editor intends to achieve the News of Specific Category Edited goal by means of the Edit News of Specific Category MacroPlan. However, to edit the news the Editor has to request the Photographer-Reporter to perform the Produce News Article of Specific Subject service. The Photographer-Reporter performs the requested 
service because it does not conflict with the achievement of the News Article Produced goal. Hence, both the requested service and the goal achievement are accomplished by means of the Contact News Agencies MacroPlan.



Fig. 7. MAS Architectural Diagram for the e-News System

\subsection{Social Pattern Selection and Application}

The last step in the detailed design phase is to select and apply social patterns to refine the architectural design of the e-News system (Fig. 7). One of the key challenges is to choose the proper social pattern to be applied to a system architectural design. One can analyze the template (e.g. [17]) that describes several features of each social pattern to address a specific requirement. For example, the e-News System has the Availability requirement, i.e., the system has to ensure easier recovery of the system if some agent in the system stops running. This requirement could not be shown in the 
case study because we did not present the requirements models of the e-News System. However, the interested reader can find it in [23].

Analyzing the template of several patterns, we have concluded that the most suitable pattern to address the Availability requirement is the Matchmaker, since it enables the search for another agent to replace the one that has stopped. To apply a social pattern, we need to weave the Matchmaker pattern with the architectural design of e-News system. To achieve this we need to instantiate each model role present in the structure of the pattern (e.g., IClient, IProvider). For example, the following bindings represent the instantiations of the model roles present in the Matchmaker SAPS (Fig. 6) for the e-News system:

1. Bind IClient to Editor

2. Bind IProvider to Photographer-Reporter

3. Bind IPerform Service to Produce News Article of Specific Subject

4. Bind IProviderlplan.laction to Photographer-Reporter.ContactNewsAgencies .getGuideline

5. Bind IClient.Iplan.Iaction to Editor.EditNewsSpecificCategory. provideSpecificSubjectGuideline

6. Bind IProvider.Iplan.|action to Photographer-Reporter.ContactNewsAgencies. produceNewsArticle

7. Bind ILocate Provider to Locate Photographer-Reporter

8. Bind IMatchmaker to Directory Facilitator

Observe that the Matchmaker role has been instantiated to Directory Facilitator because we have chosen JADE as the target agent implementation environment.

Instantiating the model roles of the pattern (Fig. 6) for the e-News model elements (Fig. 7), we obtain the pattern applied to the problem (Fig. 8). Thus, we applied the structure of the pattern to the architectural diagram of the e-News System through model roles instantiation (the bindings shown above), which can be easily automated by a CASE tool. Note that the Matchmaker pattern is refining the Editor and Photographer-Reporter AgentRoles by adding the pattern-specific concerns (ports, connectors, dependums, goals, plans). Thus, an agent playing the Editor AgentRole can search for a Photographer-Reporter at run time, as well as an agent playing the Photographer-Reporter AgentRole can publish its services in the DF's Yellow Pages. It ensures the decoupling among agents, because if a Photographer-Reporter contacted by an Editor stops running, for example, the Editor may replace that PhotographerReporter by requesting to the DF to locate another one in his yellow pages.

\subsection{The Partial AspectJ Code for the e-News System}

In this section we show how the Matchmaker Pattern introduced in Fig. 6 can be codified using the AspectJ environment. To achieve this, we use the mapping guidelines presented in section 4.3. According to these guidelines, the MacroPlans Register Services and Request Provider Identification become aspects in AspectJ. The ComplexActions to subscribe, unsubscribe and requestProviderID become advices in its corresponding aspects. The AgentRoles IProvider and IClient are the agents affected by the aspects, i.e., the Photographer-Reporter and Editor agents. The notes become pointcuts and composition rules in their corresponding aspects. 


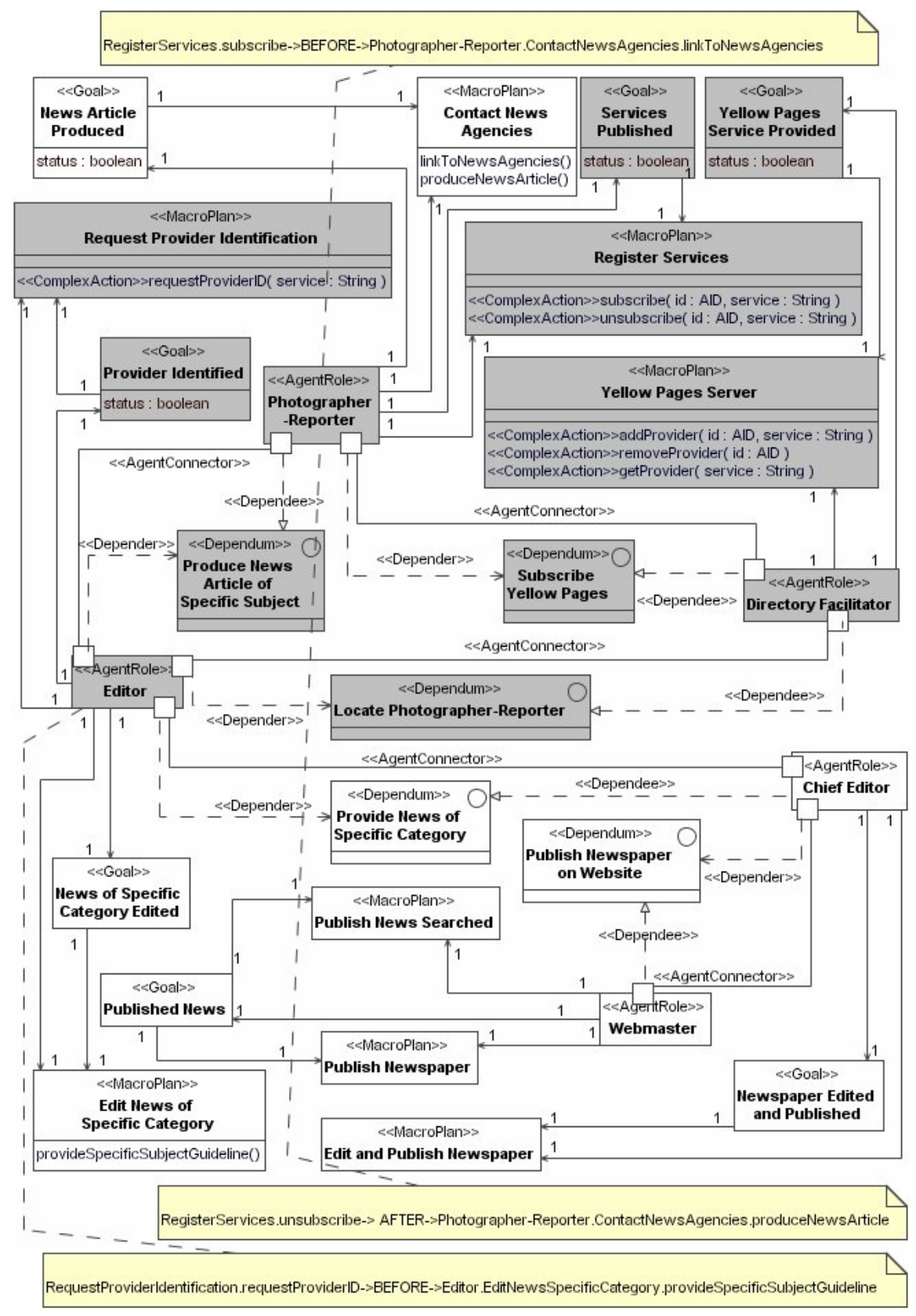

Fig. 8. MAS Architectural Diagram weaved with the SAPS

For example, in Fig. 9 we present the RegisterServices aspect weaved with the PhotographerReporter agent in the e-News System. The code after the comments "// Register..." and "// Unregister..." are the ones presented in Fig. 3 and Fig. 4, respectively. 




Fig. 9. AspectJ Code for RegisterServices Aspect

Fig. 10 presents the RequestProviderIdentification aspect weaved with the Editor agent in the e-News System. The code after the comments "// Update..." is the one presented in Fig. 5. Due to lack of space we could not illustrate the use of the mapping guidelines for the JADE environment, which can be found in [23].



Fig. 10. AspectJ Code for RequestProviderIdentification Aspect

\section{Related Work}

Aspect-oriented modeling requires the use of a higher-level aspect model that addresses the aspect-oriented programming concepts at a preliminary design stage (avoiding language specific details), and allows the designer to work at a more abstract level during software construction and evolution. Aspect-Oriented Design Model (AODM) [9] enhances the existing UML specification with aspect-oriented concepts that reproduces the crosscutting characteristics of the AspectJ language. The aSideML [8] is an aspect-oriented modeling language based on the UML that provides notation, semantics and rules for specifying aspects and crosscutting at the design level of OO systems. Composition patterns [25] is an approach that handles crosscutting concerns at design level by means of templates. However, these approaches do not address MAS directly.

It is argued in [26] that the design and implementation of agent internal architecture concerns (e.g. interaction, adaptation, autonomy, knowledge, collaboration, roles, learning and mobility) tend to affect or crosscut many classes of the system design and code, including those representing the basic agent functionality. To address this issue, [26] proposes an aspect-oriented approach to support the separate handling and modularization of MAS specific concerns. The proposed approach encourages the separate handling of agent properties, and provides a disciplined scheme for their composition. In our approach, we are concerned with the separation of social patterns 
concerns since we consider the agents already built-in (with the agent properties, such as autonomy, interaction, etc.) and supported by some agent implementation platform.

In [17], we have extended the aSideML [8] to incorporate agency features to be used in both the separation of crosscutting concerns in MAS and its latter weaving with the system agents. However, this approach becomes a little complex because we extended the aSideML by using agency features, architectural features and model roles. On the other hand, our current work is much simpler since to model crosscutting concerns of social patterns in MAS, we have created an agency metamodel and specialize it by using model roles.

\section{Conclusion and Future Work}

This work presents an approach to separate crosscutting concerns in MAS. The contribution is threefold: (i) a notation to describe the social patterns concerns separately from MAS functional modules (i.e. agent roles); (ii) a systematic way for weaving the social patterns concerns with the agent roles concerns; and (iii) guidelines to implement a system specified according to our approach using the integration of JADE and AspectJ. In doing so, we envisage several benefits for MAS design and implementation, such as improving concerns modularity, since social patterns concerns are localized into aspects while the application concerns are localized into agent roles. Consequently, this improves software reusability and maintainability.

Future work includes performing an empirical evaluation of the benefits of using aspects in the implementation of social patterns. Firstly, we need to implement MAS using only JADE, then we use the integration of JADE and AspectJ. Finally, we assess the degree of modularity of MAS in each case and compare the results. Moreover, we intent to develop a tool to support both the proposed notation and the generation code for JADE and AspectJ, as well as to apply our approach in other case studies.

\section{Acknowledgements}

This work was supported by several research grants (CNPq Proc. 142248/2004-5 \& CAPES/GRICES Proc. 129/05).

\section{References}

1. Giorgini, P., Kolp, M., Mylopoulos, J., Castro, J.: Tropos: A Requirements-Driven Methodology for Agent-Oriented Software. In: Agent-Oriented Methodologies pp. 20-45 (2005)

2. Kolp, M., Do, T., Faulkner, S., Hoang, H.: Introspecting Agent Oriented Design Patterns. In: Advances in Soft. Eng. and Knowledge Engineering, Vol. III. World Publishing (2005)

3. Noda, N., Kishi, T.: Implementing Design Patterns Using Advanced Separation of Concerns. In: OOPSLA'01, Workshop on ASoC in OO Systems, USA (2001)

4. Kiczales, G., Lamping, J., Mendhekar, A., Maeda, C., Lopes, C., Loingtier, J., Irwin, J.: Aspect-Oriented Programming. In: ECOOP'97, Finland, Springer, Heidelberg (1997)

5. Elrad, T., Filman, R., Bader, A.: Aspect-Oriented Programming: Introduction. In: Communications of the ACM 44(10), 29-32 (2001) 
6. Odell, J., Parunak, H., Bauer, B.: Extending UML for agents. In: AOIS'00 at the 17th National Conference on AI, Austin, TX, USA, pp. 3 - 17, iCue Publishing (2000)

7. Silva, V., Lucena, C.: From a Conceptual Framework for Agents and Objects to a MultiAgent System Modeling Language. In: JAAMAS'04, vol. 9(1-2), pp. 145-189. Kluwer, Dordrecht (2004)

8. Chavez, C., Garcia, A., Kulesza, U., Sant'Anna, C., Lucena, C.: Taming Heterogeneous Aspects with Crosscutting Interfaces. In: J. of the Brazilian Computer Society 12, 1 (2006)

9. Stein, D.: An Aspect-Oriented Design Model Based on AspectJ and UML. Master Thesis. University of Essen (2002)

10. Silva, C., Araújo, J., Moreira, A., Castro, J., Tedesco, P., Alencar, F., Ramos, R.: Modeling Multi-Agent Systems using UML. In: SBES'06, Brazil, pp. 81 - 96 (2006)

11. Kim, D., France, R., Ghosh, S., Song, E.: Using Role-Based Modeling. Language (RBML) as Precise Characterizations of Model Families. In: IEEE ICECCS'02, USA (2002)

12. France, F., Kim, D., Ghosh, S., Song, E.: A UML-Based Pattern Specification Technique. In: IEEE Transactions on Software Engineering 30(3), 193-206 (2004)

13. Bellifemine, F., Caire, G., Poggi, A., Rimassa, G.: JADE - A White Paper. In: Special issue on JADE of the TILAB Journal EXP (2003)

14. OMG: Unified Modeling Language (UML): Superstructure. Version 2.0, Available: (2005) www.omg.org/docs/formal/05-07-04.pdf

15. Hayden, S., Carrick, C., Yang, Q.: Architectural design patterns for multiagent coordination. In: Agents'99, Seattle, USA (1999)

16. Woods, S., Barbacci, M.: Architectural Evaluation of Collaborative Agent-Based Systems. Technical Report, CMU/SEI-99-TR-025, Carnegie Mellon University, USA (1999)

17. Silva, C., Castro, J., Araújo, J., Moreira, A., Alencar, F., Ramos, R.: Separation and Modularization of Crosscutting Social Patterns in Detailed Architectural Design. In: CAiSE'06 Forum, Tudor, Luxembourg (2006)

18. Shaw, M., Garlan, D.: Software Architecture: Perspectives on an Emerging Discipline. Prentice-Hall, Englewood Cliffs (1996)

19. Yu, E.: Modelling Strategic Relationships for Process Reengineering. Ph.D Thesis, Department of Computer Science, University of Toronto, Canada (1995)

20. Mylopoulos, J., Kolp, M., Castro, J.: UML for agent-oriented software development: The Tropos proposal. In: UML'01, Toronto, Canada (2001)

21. Gamma, E., Helm, R., Johnson, R., Vlissides, J.: Design Patterns: Elements of Reusable Object-Oriented Software. Addison-Wesley, London (1995)

22. Araújo, J., Whittle, J., Kim, D.: Modeling and Composing Scenario-Based Requirements with Aspects. In: RE'04, Kyoto, Japan (2004)

23. Silva, C.: Agent Pattern Specifications. Technical Report, Available: cin.ufpe.br/ ctlls/APS.pdf

24. Silva, C., Castro, J., Tedesco, P., Silva, I.: Describing Agent-Oriented Design Patterns in Tropos. In: Brazilian Symposium of Software Engineering (SBES'05), Brazil. pp. 10-25 (2005)

25. Clarke, S., Walker, R.: Composition Patterns: An Approach to Designing Reusable Aspects. In: ICSE'01, Toronto, Canada. pp. 12 - 19 (2001)

26. Garcia, A.: From Objects to Agents: An Aspect-Oriented Approach. PhD Thesis, Computer Science Department, PUC-Rio, Rio de Janeiro, Brazil (2004) 